randomisation and the funding of prehospital critical care for OHCA.

Method We aimed to answer the following questions: What are stakeholders' priorities for prehospital research? What are stakeholders' views on randomisation of prehospital critical care? How do stakeholders consider allocation of resources in prehospital care? We undertook a qualitative framework analysis of interviews and focus group with five key stakeholder groups: patients and public, air ambulance charities, ambulance service commissioners, prehospital researchers and prehospital critical care providers.

Results Despite sharing a common appreciation of the concepts of scientific enquiry, fairness, and beneficence, the five relevant stakeholder groups displayed divergent views of research and funding strategies regarding the intervention of prehospital critical care for the condition of OHCA. The reasons for this divergence could largely be explained through the different personal experiences and situational contexts of each stakeholder group. Many aspects of the strategies suggested by the stakeholder groups only partially aligned with principles of traditional evidence-based medicine, but were held with strong conviction.

Conclusion Analysis of the views of five stakeholder groups regarding research and the funding of prehospital critical care for OHCA revealed shared values, but a variety of different strategies to achieve these. This knowledge can help researchers in similar fields in the planning and presentation of their research, to maximise impact on decision making.

Conflict of interest Johannes von Vopelius-Feldt and Jonathan Benger work as prehospital physicians with the Great Western Air Ambulance.

Funding This work is funded by a National Institute for Health Research (NIHR) doctoral research fellowship for Johannes von Vopelius-Feldt (DRF-2015-08-040). The funder is not involved in the design of the study or collection, analysis and interpretation of data, or in writing the manuscript. The views expressed are those of the author(s) and not necessarily those of the NHS, the NIHR or the Department of Health.

\section{SYSTEMATIC REVIEW OF THE EFFECTIVENESS OF PREHOSPITAL CRITICAL CARE FOLLOWING OUT-OF- HOSPITAL CARDIAC ARREST}

\footnotetext{
1,2Johannes von Vopelius-Feldt*, ${ }^{2}$ Janet Brandling, 1,2Jonathan Benger. 'Academic Department of Emergency Care, University Hospitals Bristol; ${ }^{2}$ Emergency Medicine and Critical Care Research Group, University of the West of UK
}

\subsection{6/10.1136/bmjopen-2018-EMS.10}

Aim Improving survival after out-of-hospital cardiac arrest (OHCA) is a priority for modern emergency medical services (EMS) and prehospital research. Advanced life support (ALS) is now the standard of care in most EMS. In some EMS, prehospital critical care providers are also dispatched to attend OHCA. This systematic review presents the evidence for prehospital critical care for OHCA, when compared to standard ALS care.

Method We searched the following electronic databases: PubMed, EmBASE, CINAHL Plus and AMED (via EBSCO), Cochrane Database of Systematic Reviews, DARE, Cochrane Central Register of Controlled Trials, NHS Economic Evaluation Database, NIHR Health Technology Assessment Database, Google Scholar and ClinicalTrials.gov. Search terms related to cardiac arrest and prehospital critical care. All studies that compared patient-centred outcomes between prehospital critical care and ALS for OHCA were included.

Results The review identified six full text publications that matched the inclusion criteria, all of which are observational studies. Three studies showed no benefit from prehospital critical care but were underpowered with sample sizes of 1028 to 1851 . The other three publications showed benefit from prehospital critical care delivered by physicians. However, an imbalance of prognostic factors and hospital treatment in these studies systematically favoured the prehospital critical care group.

Conclusion Current evidence to support prehospital critical care for OHCA is limited by the logistic difficulties of undertaking high quality research in this area. Further research needs an appropriate sample size with adjustments for confounding factors in observational research design.

Conflict of interest Johannes von Vopelius-Feldt and Jonathan Benger work as prehospital critical care physicians with the Great Western Air Ambulance.

Funding This work is funded by a National Institute for Health Research (NIHR) doctoral research fellowship for Johannes von Vopelius-Feldt (DRF-2015-08-040). The funder is not involved in the design of the study or collection, analysis and interpretation of data, or in writing the manuscript. The views expressed are those of the author(s) and not necessarily those of the NHS, the NIHR or the Department of Health.

\section{QUALITY OF BYSTANDER CPR BY LAY FIRST RESPONDERS: TRAINING VERSUS REAL-WORLD USE OF A NOVEL CPR FEEDBACK DEVICE IN SINGAPORE}

${ }^{1}$ AE White*, ${ }^{1}$ W Wah, ${ }^{1}$ NAM Jalil, ${ }^{1} \mathrm{NJ}$ Lum, ${ }^{1} \mathrm{Ng}$ EKX, ${ }^{2} \mathrm{PHJ}$ Kua, ${ }^{3,4} \mathrm{MEH}$ Ong. ${ }^{1}$ Unit for Prehospital Emergency Care, Singapore General Hospital, Singapore; ${ }^{2}$ Department of Emergency Medicine, KK Women's and Children's Hospital, Singapore; 'Department of Emergency Medicine, Singapore General Hospital, Singapore; ${ }^{4}$ Health Services and Systems Research, Duke-NUS Graduate Medical School, Singapore

\subsection{6/10.1136/bmjopen-2018-EMS.11}

Aim Data on the quality of lay person CPR during emergencies are sparse. We present compression quality data derived from use of a novel CPR feedback device during actual cases prior to ambulance arrival.

Method The credit-card sized CPRcardTM device provided visual indication of compression depth and rate in real-time, and stored the data. Median rate, depth; proportion within targets (100-120/minute; depth:4-6 cm); and flow-time were used to determined compression quality. Bystanders' emergency performances were compared to their training performances.

Results Median depth during emergencies vs trainings was $39 \mathrm{~mm} \quad(95 \% \mathrm{CI}: 30$ to $49 \mathrm{~mm}, \mathrm{p}=0.028)$ vs $55 \mathrm{~mm}$ (95\% CI: 50 to $57 \mathrm{~mm}, \mathrm{p}=0.028)$; and median rates were $114 \mathrm{cpm}$ (95\% CI: 109 to $120 \mathrm{cpm}, \mathrm{p}=0.104)$ vs $109 \mathrm{cpm}$ (95\% CI: 105 to $112 \mathrm{cpm}, \mathrm{p}=0.104)$. Of total emergency vs training delivered compressions, 6\% (95\% CI: $0 \%$ to $49 \%$, $\mathrm{p}=0.008)$ vs $63 \% \quad(95 \% \mathrm{CI}: 56$ to $90 \%, \mathrm{p}=0.008)$ were within target depth; $54 \%$ (95\% CI: $32 \%$ to $79 \%, p=0.028$ ) vs $94 \%$ (95\% CI: 81 to $97 \%, p=0.028$ ) were within target rate. Of the lay bystanders' during emergencies vs trainings, 4 $(50 \%, \mathrm{p}=0.398)$ vs $5(71 \%, \mathrm{p}=0.398)$ met both compression and depth targets. Emergency vs training compression flowtime was $95 \%$ (95\% CI: $85 \%$ to $99 \%, p=0.099)$ vs $100 \%$ (95\% CI: 96 to 100\%, $\mathrm{p}=0.099$ ), respectively. Lay bystanders overall reported positive experience using the card but some 